\title{
TEST RESULTS FOR ENTRY GUIDANCE METHODS FOR REUSABLE LAUNCH VEHICLES draft
}

\author{
John M. Hanson \\ Aerospace Engineer, NASA Marshall Space Flight Center, Huntsville, AL \\ Robert E. Jones \\ Senior Engineer, Sverdrup Technology, Inc., Huntsville, AL
}

\begin{abstract}
$\underline{\text { Abstract }}$
There are a number of approaches to advanced guidance and control (AG\&C) that have the potential for achieving the goals of significantly increasing reusable launch vehicle (RLV) safety and reliability, and reducing the cost. This paper examines some approaches to entry guidance. An effort called Integration and Testing of Advanced Guidance and Control Technologies (ITAGCT) has recently completed a rigorous testing phase where these algorithms faced high-fidelity vehicle models and were required to perform a variety of representative tests. The algorithm developers spent substantial effort improving the algorithm performance in the testing. This paper lists the test cases used to demonstrate that the desired results are achieved, shows an automated test scoring method that greatly reduces the evaluation effort required, and displays results of the tests. Results show a significant improvement over previous guidance approaches. The two best-scoring algorithm approaches show roughly equivalent results and are ready to be applied to future reusable vehicle concepts.
\end{abstract}

\section{Introduction}

Advanced guidance and control has a significant potential to increase the safety of future reusable launch vehicles, as well as to reduce the cost of performing guidance and control analysis, both in the design and in the operational phases. This potential has been documented elsewhere ${ }^{1}$. The Advanced Guidance and Control Project, which was supported by the NASA X-33 Program Office, had as its purpose to develop and test some of the potential methods. The testing was to be in a high-fidelity simulation, against a number of stressing conditions, in order to discern the most flexible approaches.

This paper describes some advanced approaches for entry guidance. We summarize an initial plase of testing performed to examine the various methods. Some lessons were learned from the initial phase of testing. Some of the algorithms performed well, but for the most part the methods were not ready to address all the RLV needs. We planned a second phase of testing to more completely examine the performance of the algorithms versus the safety and cost requirements. This paper includes a description of the test cases for the second phase of testing. This paper also describes an automated method of scoring the algorithm performance that leads to a significant reduction of effort. We include final results of the second phase of testing at the end of the paper. The testing and algorithm improvement work were supported by the Space Launch Initiative Program. This paper is the first in what is hoped to be a series of papers that examines tests of the various algorithms needed for a complete advanced guidance and control architecture.

\section{First Phase of Testing}

An original goal in this effort was to include as many approaches as possible within the resources of the effort, with an eye toward not missing what may be the best approach. The methods had to be openly available (not proprietary), and available with a relatively small budget. This led to an emphasis on university grants and in-house efforts.

A test series was conducted in September 2000. Four entry guidance methods were tested. The test environment was the X-33 Marshall Aerospace Vehicle Representation in C (MAVERIC) simulation ${ }^{2}$. MAVERIC was the only high-fidelity end-to-end simulation used for X-33 design. The X33 was planned to fly a number of sub-orbital test flights, so these tests encompassed primarily ascent followed immediately by entry on sub-orbital trajectories. The sub-orbital flights should be viewed as representing different downrange aborts for a RLV. These tests are particularly stressing for entry guidance, because the duration of the entry phase is short, and because the entry guidance has to remove dispersions that result from the ascent phase. Additional tests were run for entry from various orbits, using the same simulation and vehicle models, 
with differing crossrange requirements and heat constraints.

Tests during Phase 1 included different nominal missions, engine-out aborts, Monte Carlo dispersion runs for both nominal missions and aborts, and significant engine over and under performance. Failures and mis-modeling not associated with the propulsion system were not explicitly considered for this Phase 1 testing. Algorithm size, speed of execution, memory, complexity/effort required, and performance against a variety of criteria were all compared.

Of all the methods tested, the linear quadratic regulator entry guidance was the only one that performed quite well. In all cases, it became clear that more work was necessary to develop the algorithms to their full potential, so that they would successfully fly the various test cases. This led to the definition of a second set of tests, as described below, and to more work on the algorithms, as described in the references.

\section{Methods to be Examined}

The work in this paper continues from work first described in Reference 2. The following describes the different methods involved in this integration and testing.

- Baseline guidance. This method is like the Shuttle entry guidance in that it tracks a nominal drag versus energy profile for longitudinal guidance and uses a heading error corridor to trigger periodic bank sign reversals for lateral guidance. It was the baseline entry guidance for $X-33$. This guidance was used for a portion of the tests, for comparison purposes. It was not used for the complete set of tests because of the time it would have taken to set it up and tune it properly for all tests. Add a bit more detail from the Boston paper with reference.

- Linear quadratic regulator (LQR). This method ${ }^{3}$ has performed very well in test. The algorithm is of the reference profile tracking type. The reference profile consists of reference states, range to go, altitude, and flight path angle, and reference controls, bank angle and angle of attack, versus energy. A linear control law using state feedback is used with energy-scheduled gains. The gains are obtained offline using Matlab's steady state linear quadratic regulator function. Lateral trajectory control is obtained through bank sign reversals chosen based on a heading error corridor. The algorithm is not quite as flexible as methods with on-board trajectory re-generation capability, but it is very robust with respect to varying initial conditions. The guidance gains are for the most part trajectory-independent.

- Predictor-corrector. This method ${ }^{4}$, called Eguide, chooses parameters using a Newton procedure and numerical integration to obtain a desirable trajectory on-board for the actual flight conditions. Eguide follows a reference heat rate early in entry. A total of four parameters are chosen for entry--reference heat rate, initial bank angle, bank angle rate, and the time to switch from heat rate tracking to targeting the TAEM interface. TAEM, or Terminal Area Energy Management, is the flight phase that occurs after entry and is focused on setting up the landing phase. For this study, the TAEM interface is at about $2500 \mathrm{fps}$ relative speed, $30 \mathrm{~nm}$ from the landing area, and at about $95,000 \mathrm{ft}$ altitude. After the high heating region is passed, Eguide uses initial bank and bank rate to target the TAEM interface. EGuide contains a planning stage and also functions as guidance with a combination of a predictor/corrector and a profile-follower using the $L Q R$ guidance just described. This method results in very smooth bank angle and angle of attack commands, and a smooth altitude profile. A bank reversal is chosen to satisfy the lateral motion necessary to reach the TAEM interface.

- A trajectory design method that uses quasiequilibrium glide, combined with a predictorcorrector method, to design a trajectory for entry ${ }^{5}$. The use of equilibrium glide enables the inequality constraints to be observed. The entry trajectory design problem is decomposed into two sequential one-parameter search problems. The 3-dimensional trajectory is derived (longitudinal and lateral motion), in order for the TAEM interface to be successfully reached. First, the motion for the initial entry into the atmosphere and the longitudinal profile for the pre-TAEM portion are found. Next, the method estimates the longitudinal profile for the quasiequilibrium glide phase. Integrating the equations of motion enables the trajectory to converge and to follow the correct vehicle dynamics. The combined longitudinal motion satisfies the constraints and end conditions. A bank reversal is chosen to satisfy the lateral motion requirements. A profile-following guidance flies the trajectory, focusing on following all the trajectory parameters ${ }^{6}$.

- An entry trajectory design and guidance procedure based on extension of the Shuttle 
trajectory design methods to three dimensions?. The method is called EAGLE, for Evolved Acceleration Guidance Logic for Entry. The planning algorithm generates reference drag acceleration and lateral acceleration profiles, along with the reference state and bank angle profiles. EAGLE chooses a drag profile using a 3-segment linear spline fit for a profile that fits within the drag versus velocity constraints and gives the correct value for downrange distance. The lateral motion is determined through a bank reversal chosen to minimize the final crossrange error. An iteration is used to improve the length and curvature estimates. A feedback linearization control is used to track the reference drag and heading profiles.

\section{Test Cases and Test Criteria}

For the second phase of tests, we included many of the first set of tests again, since the methods did not in most cases perform satisfactorily. We also added tests for various failure and mis-modeling cases that seemed appropriate. The test environment was a newer version of MAVERIC that models the X-33 vehicle in more detail and automates some of the test processes required. A list of the test cases follows in Table 1. Table 2 shows the motivation for each set of tests. Table 3 lists the dispersions modeled for the Monte Carlo runs.

\section{Table 1. Phase 2 Test Series}

DOF: D̄egrees of Freedom; MiCD: Mionte Carlo Dispersions; PPO: Power Pack Out (Engine Failure, time of failure indicated); Michael (nominal) and Ibex (low energy) are X-33 landing sites; Mich10al and 10dl are different X-33 missions (10d1 is a higher-energy flight); seed indicates whether a new random number was used to start certain test cases. All environments are for the month of April unless noted. ISS is International Space Station; LEO is low Earth orbit.

ENTRY GUDANCE TEST SERIES

\begin{tabular}{|c|c|c|}
\hline Test Number \& Description & DOF & \# Runs \\
\hline 1) Mich10al & 6 & $100 \mathrm{MCD}$ \\
\hline 2) Mich10al, February environment, different random seed & 6 & $200 \mathrm{MCD}$ \\
\hline 3) Mich10d1 & 6 & $100 \mathrm{MCD}$ \\
\hline 4) Mich10d1, August environment, different random seed & 6 & $200 \mathrm{MCD}$ \\
\hline 5) Michl0al, PPO time $50 \mathrm{sec}$ (early abort to Michael) & 3 & $100 \mathrm{MCD}$ \\
\hline 6) Michl0al, PPO time $60 \mathrm{sec}$ & 3 & $100 \mathrm{MCD}$ \\
\hline 7) Mich10al, PPO time $112 \mathrm{sec}$ & 3 & $100 \mathrm{MCD}$ \\
\hline 8) Michl0al, PPO time 40 sec (early to Ihex), different random seed & 3 & $200 \mathrm{MCD}$ \\
\hline 9) Michl0dl, PPO time $38 \mathrm{sec}$ (early to Ibex), different random seed & 3 & $200 \mathrm{MCD}$ \\
\hline 10) Mich10al, +4 sigma thrust dispersion from ascent & 3 & 1 \\
\hline 11) Mich10al, +6 sigma thrust dispersion from ascent & 3 & 1 \\
\hline 12) Mich10al, -12 sigma thrust dispersion from ascent & 3 & 1 \\
\hline $\begin{array}{l}\text { 13) } 51.6 \text { deg. ISS orbit entry, low crossrange, high peak heat rate limit, input profile to guidance is from } \\
\text { this trajectory's design. }\end{array}$ & 3 & $100 \mathrm{MCD}$ \\
\hline 14) 51.6 deg. ISS orbit entry, high right crossrange, high peak heat rate limit, input profile from 13 . & 3 & $100 \mathrm{MCD}$ \\
\hline 15) 51.6 deg. ISS orbit entry, high left crossrange, high peak heat rate limit, input profile from 13. & 3 & $100 \mathrm{MCD}$ \\
\hline $\begin{array}{l}\text { 16) } 51.6 \text { deg. ISS orbit entry, low crossrange, low peak heat rate limit, input profile from this trajectory's } \\
\text { design. }\end{array}$ & 3 & $100 \mathrm{MCD}$ \\
\hline 17) $51.6 \mathrm{deg}$. ISS orbit entry, high right crossrange, low peak heat rate limit, input profile from 16. & 3 & $100 \mathrm{MCD}$ \\
\hline 18) 51.6 deg. ISS orbit entry, high left crossrange, low peak heat rate limit, input profile from 16. & 3 & $100 \mathrm{MCD}$ \\
\hline 19) 28.5 deg. LEO orbit entry, low crossrange, low peak heat rate limit, input profile from 16. & 3 & $100 \mathrm{MCD}$ \\
\hline 20) $28.5 \mathrm{deg}$. LEO orbit entry, high right crossrange, low peak heat rate limit, input profile from 16 . & 3 & $100 \mathrm{MCD}$ \\
\hline 21) $28.5 \mathrm{deg}$. LEO orbit entry, high left crossrange, low peak heat rate limit, input profile from 16. & 3 & $100 \mathrm{MCD}$ \\
\hline 22) Mich 10a 1, aerosurface failure result: angle of attack limited to $5 \mathrm{deg}$. less that nominal entry value. & 3 & 1 \\
\hline 23) Mich10al, aerosurface failure result: angle of attack and bank rates limited to $2 \mathrm{deg} / \mathrm{sec}$. maximum. & 3 & 1 \\
\hline 24) Mich10dl, aerosurface failure: angle of attack limited to $5 \mathrm{deg}$. less that nominal entry value. & 3 & 1 \\
\hline $\begin{array}{l}\text { Mich10dl, aerosurface failure: angle of attack limited to } 5 \text { deg. less that nominal entry value, and angle } \\
\text { of attack and bank rates limited to } 2 \text { deg./sec. Maximum }\end{array}$ & 3 & 1 \\
\hline $\begin{array}{l}\text { Mich 10a1, unknown to guidance, first flight aerodynamics mis-modeling: aerodynamic lift coef. } 20 \% \\
\text { less than vehicle database model. }\end{array}$ & 3 & 1 \\
\hline $\begin{array}{l}\text { 27) Mich10al, unknown to guidance, first flight aerodynamics mis-modeling: aerodynamic lift coef. } 20 \% \\
\text { more than vehicle database model. }\end{array}$ & 3 & 1 \\
\hline 28) Mich10al, unknown to guidance, first flight aerodynamics mis-modeling: aerodynamic lift coef. $20 \%$ & 3 & 1 \\
\hline
\end{tabular}


Table 2. Motivation for the Tests

\begin{tabular}{|l|l|}
\hline Tests & Motivation \\
\hline $1-4$ & Nominal X-33 missions with dispersions; robustness to dispersions \\
\hline $5-9$ & Engine failures; robustness to large off-energy cases and alternate landing sites \\
\hline $10-12$ & Large thrust dispersions; ability to maximize probability of successful landing \\
\hline $13-21$ & $\begin{array}{l}\text { Entry from orbit; ability to adapt to different heating requirements and crossrange } \\
\text { requirements with dispersions. }\end{array}$ \\
\hline $22-25$ & Effects from failures causing a change in maneuverability \\
\hline $26-28$ & Mis-modeling of aerodynamics on first flight \\
\hline
\end{tabular}

Table 3. Dispersions

Atmospheric density: GRAM 99 atmosphere with random seed

Winds: GRAM 99 atmosphere with random seed

De-orbit Parameters

Aerodynamics

More detail will be added to this table; define GRAM, add reference?.

\section{Automated Scoring}

The Phase 1 test evaluation involved a number of guidance and control experts reviewing the results (both graphical and numerical) and determining how well the method flew the vehicle. This approach worked, but had two drawbacks: 1) It requires a large amount of engineer time for evaluation of many parameters on many tests for multiple algorithms, and 2) the final evaluation has some subjectivity in it (and could potentially result in uneven evaluation). There was a benefit to this method, however. In evaluating the methods, it became clear to the evaluators what parameters were important to them and what values of these parameters were acceptable. As a result, we were able to automate the scoring process for the Phase 2 tests.

Tests are numerically scored, and then each test is weighted, with the scores added, so that the algorithms have a final numerical score. Normalization results in a perfect score being given a value of 1.0. For each parameter to be tested, there is a weight, and these multiply that parameter's score and add into the total. Single tests (not Monte Carlo dispersions) are scored as in this example:

Normal acceleration: $0-3.5 \mathrm{~g}, 1.0-2.5 \mathrm{~g}$ means the score is $\mathbf{1 . 0}$ for normal acceleration magnitudes below $2.5,0.0$ for values above 3.5 , and linearly varying in between the two limit values. The parameter score is multiplied by the weight for that parameter (normal acceleration) and added into the total score for that test.

For Monte Carlo dispersion tests, the overall score is the average of the individual scores. A final criteria used for the entry guidance and flight control tests regards accuracy in reaching the TAEM targets. If the range, altitude, and heading angles are not sufficiently controlled in order to be able to land successfully, the test was considered a failure (score of 0 ) even if other criteria were met. Typical values used for the required accuracy at hitting the TAEM condition were $7 \mathrm{~nm}, 7000 \mathrm{ft}$, and $10 \mathrm{deg}$, respectively. If more than $10 \%$ of Monte Carlo cases fail to meet these TAEM conditions, then the entire Monte Carlo run is given a score of 0.0 . An exception to this last criteria was tests 5 and 8 . We found that the $90 \%$ success criteria resulted in all algorithms failing this test. By removing the criteria, it became possible to see the relative scores of the various algorithms and thus to have a means for comparing them.

Table 4 lists the criteria compared for each of the various tests. We plan to provide more detail in the final paper for Table 4's description of the criteria, with values and weights included. 

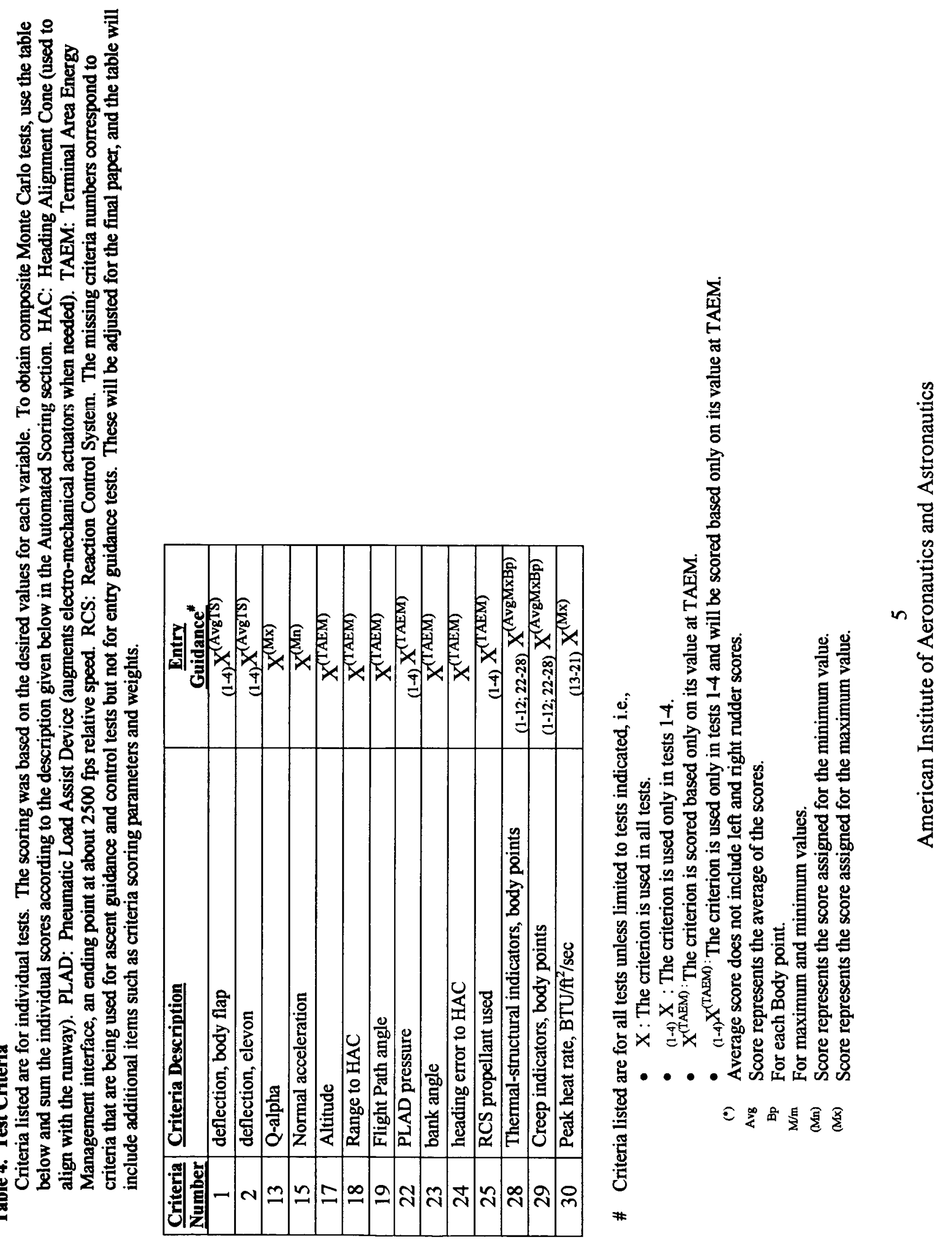


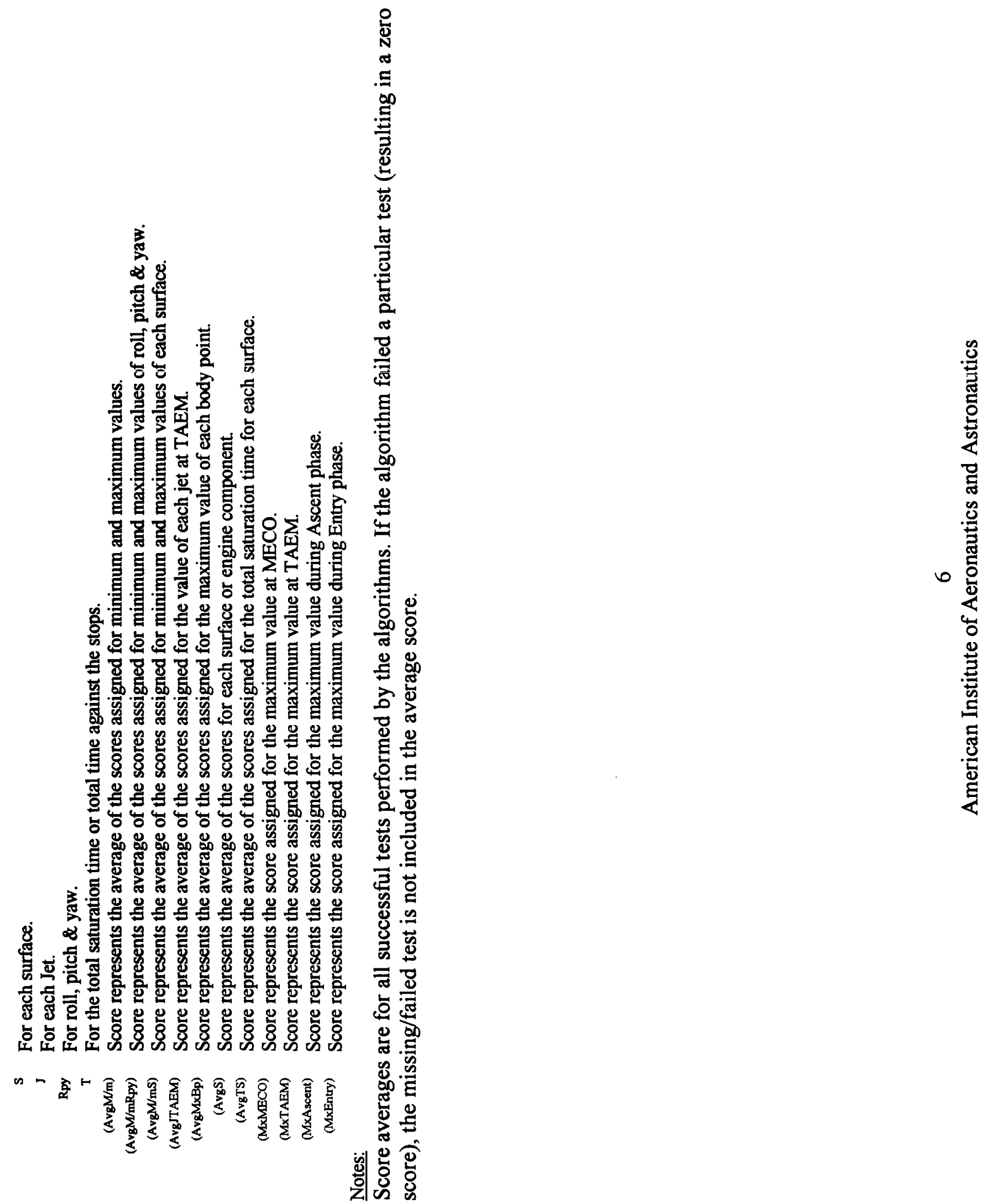




\section{Results}

Results of the tests are shown in the following figures. These tests have been performed a number of times, with the algorithm designers given a chance to correct problems in their method's performance during the process. The baseline X-33 entry guidance (Shuttle-like) is shown for comparison for tests where it is set up to fly the vehicle.

Figure 1 shows the performance for the entry guidance test cases. A score of 0.0 means that the algorithm failed the test. If more than one algorithm failed the test, a slightly negative score is used so that the reader is able to see the scores in the figure. The $\mathrm{X}-33$ baseline guidance is used for tests $1-12$ only. It is clear that it does quite poorly as compared to the other algorithms. The reason for this is clearly visible in Figure 2, which shows results from Test

Case 1. The nominal TAEM interface altitude and range are $96,000 \mathrm{ft}$ and $30 \mathrm{~nm}$ respectively. Although the baseline guidance has values generally clustered in this vicinity, the spread is significantly greater than the LQR TAEM spread, which is very tight around the nominal values. Four of the 100 cases for the baseline are off the graph and others would not be land-able. The particular failed criteria in this case is that at least $90 \%$ of cases must be within $7 \mathrm{~nm}$ of the desired TAEM range condition. Although this baseline algorithm would be completely acceptable for flying a typical Shuttle entry profile that has a long time available for removing dispersions as well as a benignly-designed trajectory, the X-33 sub-orbital trajectory was required to remove ascent dispersions on a relatively sporty entry profile in a much shorter period of time. This may be necessary in some downrange abort scenarios for an orbital vehicle.

The LQR algorithm scores fairly well across the board, although it fails some of the more difficult cases, probably due to the fact that it does not have a trajectory profile tailored more to that case. Test case 20 is a high crossrange case that LQR fails because it does not have a trajectory profile tailored to high crossrange.

The predictor-corrector (Eguide) method performs well, although it has some problems. Its capability to design smooth new profiles, combined with a robust guidance that includes the LQR after the high heating region is passed, leads to robust performance. It fails some of the toughest tests. The designer of this algorithm realized that further improvements would possibly have removed the failures. However, he decided not to continue working on the algorithm after seeing success in the other algorithms. Further work may have brought the performance of this approach up to near the others, but would have probably not yielded equal or greater performance.

The quasi-equilibrium glide approach and the EAGLE algorithm both performed extremely well. Both approaches passed all the tests and performed well on all tests. The total scores for the various algorithms, as of the final deadline for testing, are as follows: update this score

$\begin{array}{ll}\text { Drag-Energy 3D } & 94.3 \\ \text { Quasi-Equilibrium Glide } & 94.3 \\ \text { Linear Quadratic Regulator } & 83.5 \\ \text { Predictor-Corrector } & 82.3 \\ \text { Shuttle-like } & 8.4 \text { (out of a }\end{array}$

possible 50)

The drag-energy 3D approach ultimately scored $95.9 \%$ in a subsequent test, but the algorithm was submitted separately for the different X-33 missions (10al and 10dl), whereas the quasi-equilibrium glide testing is for a single algorithm.

The criteria graph (Fig. 3) shows the performance on the various criteria for each algorithm. The criteria are in Table 4. The performance is shown only for those tests that did not fail (did not score a zero on the test cases graph). This way, the reader will see information on how the method performed for the various criteria. The number of successful tests for each algorithm can be determined from the test cases graph. Since the baseline passed only three tests, its criteria numbers are not meaningful and are left out of the figure.

All of the new algorithms performed fairly well against the various criteria. There are some differences where some of the approaches are stronger than others.

\section{Summary}

This paper gives the results from testing entry guidance methods for application to future reusable launch vehicles. The methods were tested in highfidelity simulation to determine their performance with respect to nominal missions, engine-out situations, dispersions, various failures, and vehicle mis-modeling. All the new approaches significantly outperformed the Shuttle-based entry guidance design. The quasi-equilibrium glide approach and the drag-energy 3D method (EAGLE) both 
performed extremely well. We believe they are now proven to the extent that they can be expected to work well on a future vehicle application. The $L Q R$ approach, while having more difficulty with some of the tests as compared to the two top performers, is simpler computationally and would represent a good choice for a lower-risk approach.

As of this writing, the EAGLE method is not a single algorithm, but has been delivered separately for different test cases.

For future differentiation between the various approaches, an option would be to define a couple of additional tests to hopefully separate the two top performing algorithms. The tests might include running the algorithms for some scenarios that were not available to the designers, to find out how robust they are to unforeseen scenarios. Relative computational complexity is another way to compare the approaches.

Follow-on work is planned in all the other areas of advanced guidance and control, which are not as far developed as the entry guidance. Although work is progressing on all fronts, much work remains to be done. Each of the best algorithm approaches should then be integrated into an overall advanced guidance and control architecture. This architecture would be shown to meet the safety, reliability, and cost objectives.

\section{References}

1. Hanson, J., "A Plan for Advanced Guidance and Control Technology for $2^{\text {nd }}$ Generation Reusable Launch Vehicles," paper 2002-4557, 2002 AIAA GN\&C Conference, Monterey, CA, Aug 2002.

2. Hanson, J., "Advanced Guidance and Control Project for Reusable Launch Vehicles," AIAA2000-3957, Proceedings of the 2000 AIAA GN\&C Conference.

3. Dukeman, G.A., "Profile-Following Entry Guidance Using Linear Quadratic Regulator Theory," paper 2002-4457, AIAA GN\&C Conference, Monterey, CA, Aug 2002.

4. Zimmerman, C., Dukeman, G., and Hanson, J., "An Automated Method to Compute Orbital Reentry Trajectories with Heating Constraints," paper 2002-4454, AIAA GN\&C Conference, Monterey, CA, Aug 2002.

5. Shen, Z., and Lu, P., "On-Board Generation of Three-Dimensional Constrained Entry Trajectories," Journal of Guidance, Control, and Dynamics, Vol. 26, No. 1, Jan-Feb 2003.

6. Lu, P., "Regulation about Time-Varying Trajectories: Precision Entry Guidance Illustrated", Journal of Guidance, Control, and Dynamics, Vol. 22, No. 6, 1999, pp. 784-790.

7. Chen, D.T., Saraf, A., Leavitt, J.A., and Mease, K.D., "Performance of Evolved Acceleration Guidance Logic for Entry (EAGLE)," paper 2002-4456, AIAA GN\&C Conference, Monterey, CA, Aug 2002. 


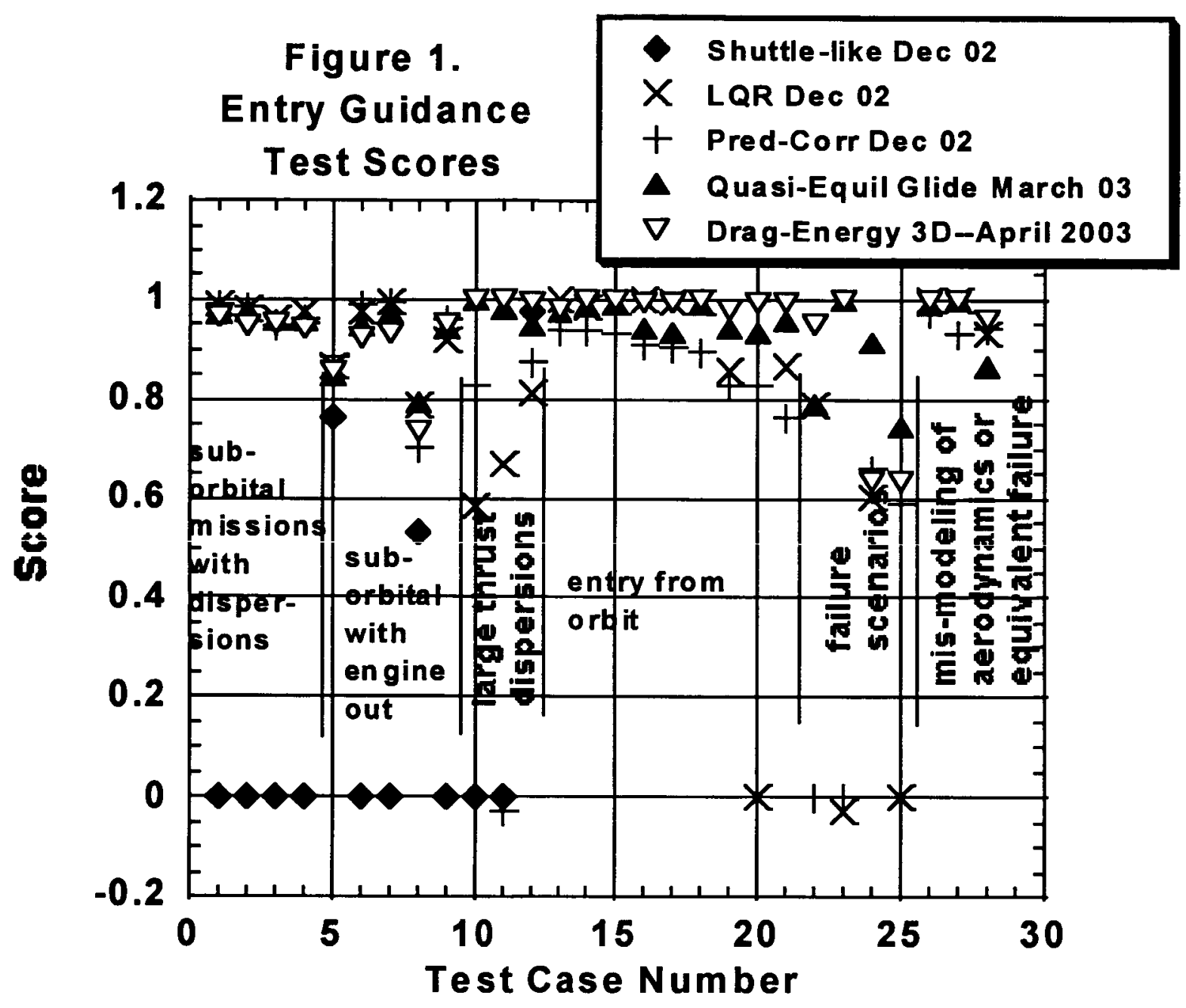


Figure 2. Altitude vs Range Comparison

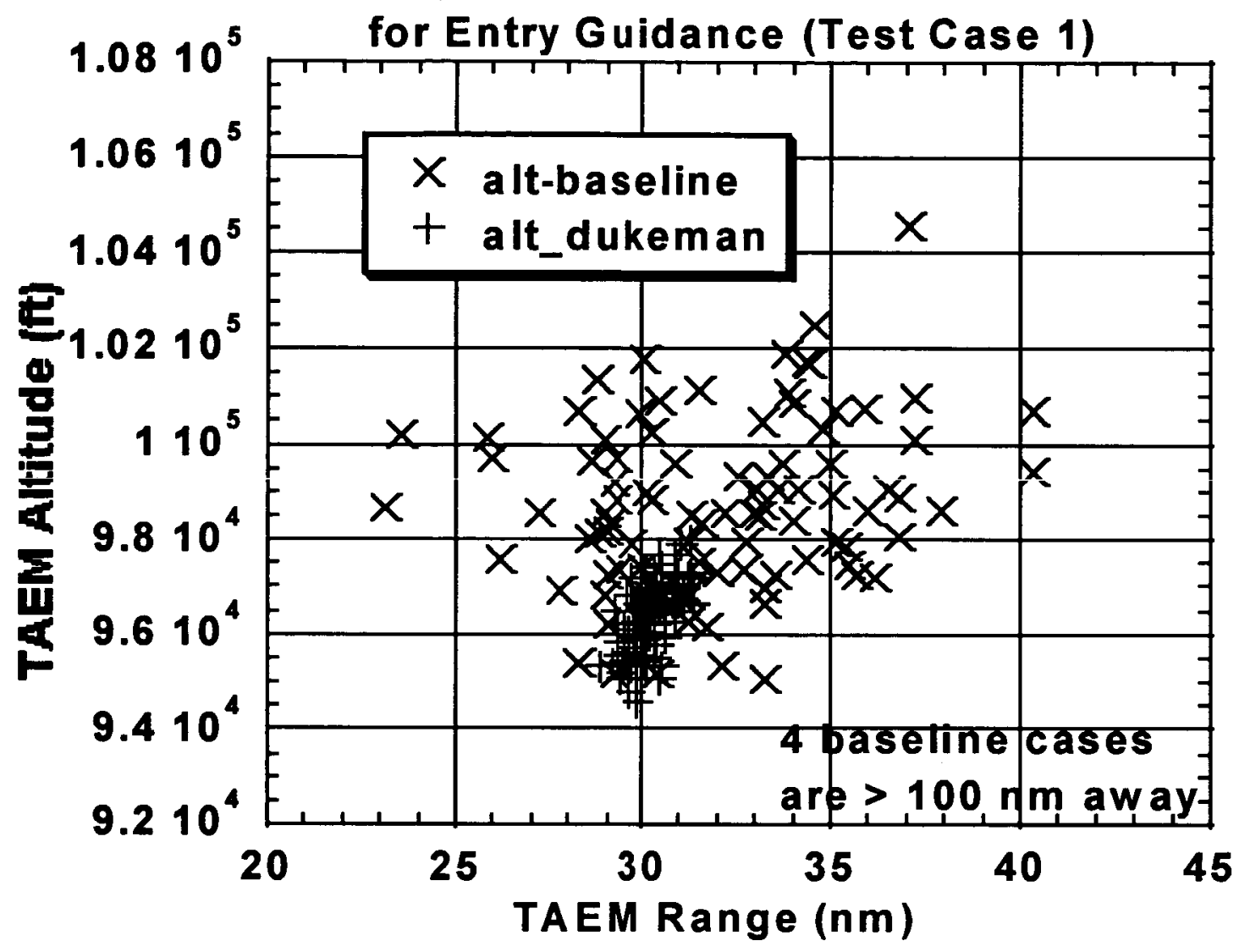




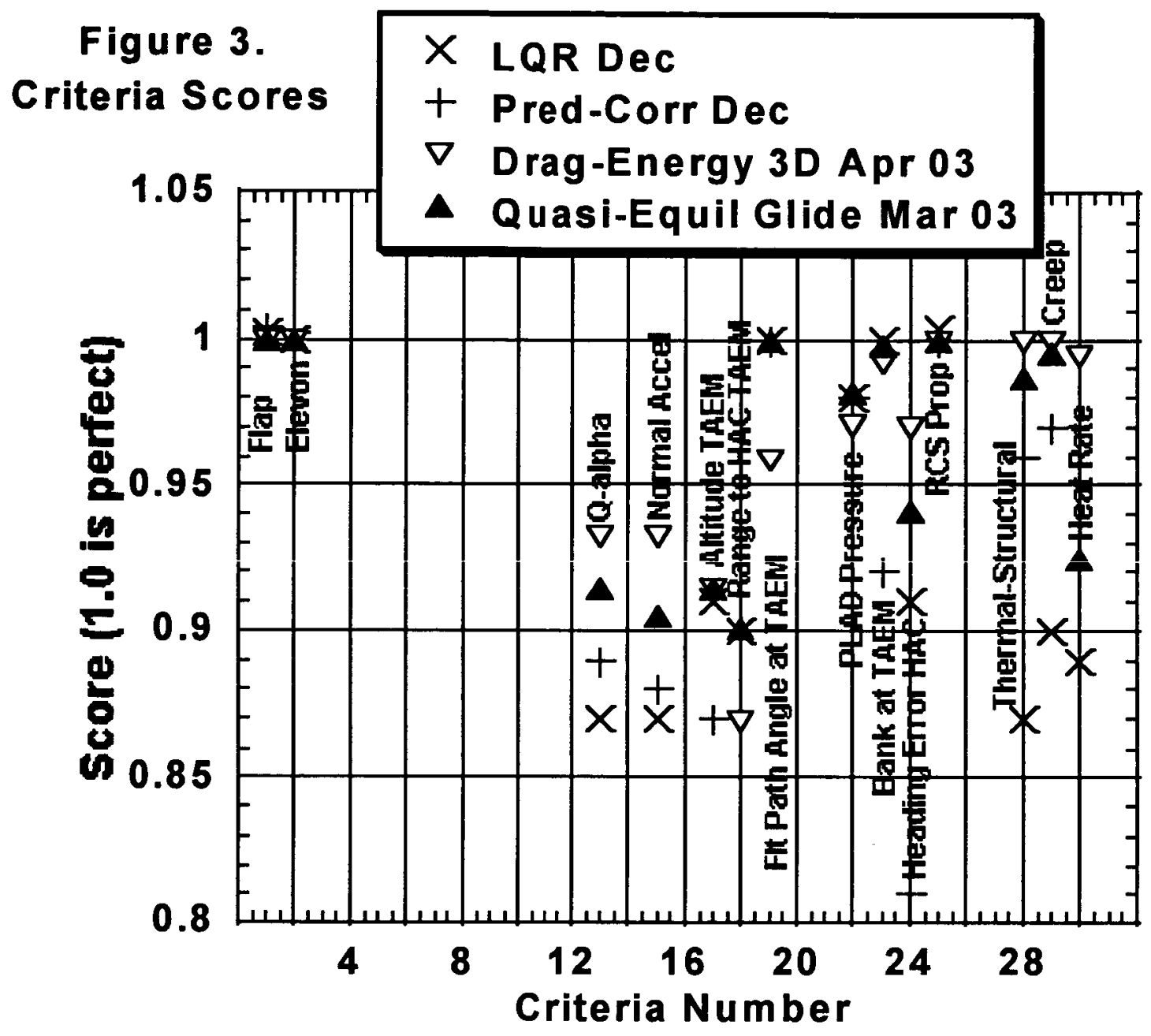

